\title{
Analysis of Promyelocytic Leukemia in Human Embryonic Carcinoma Stem Cells During Retinoic Acid-Induced Neural Differentiation
}

\author{
Khadijeh Karbalaie ${ }^{1,2}$, Sadeq Vallian ${ }^{* *}$, Liana Lachinani ${ }^{3}$, Somayeh Tanhaei ${ }^{4}$, Hossein Baharvand ${ }^{5,6^{* *}}$, \\ Mohammad Hossein Nasr-Esfahani 2** \\ ${ }^{1}$ Division of Genetics, Department of Biology, Faculty of Science, University of Isfahan, Isfahan, Iran \\ ${ }^{2}$ Department of Cellular Biotechnology, Cell Science Research Center, Royan Institute for Biotechnology, ACECR, Isfahan, Iran \\ ${ }^{3}$ Department of Cell and Molecular Biology, Cell Science Research Center, Royan Institute for Biotechnology, ACECR, Isfahan, \\ Iran \\ ${ }^{4}$ Department of Molecular Genetics, Cell Science Research Center, Royan Institute for Biotechnology, ACECR, Isfahan, Iran \\ ${ }^{5}$ Department of Stem Cells and Developmental Biology, Cell Science Research Center, Royan Institute for Stem Cell \\ Biology and Technology, ACECR, Tehran, Iran \\ ${ }^{6}$ Department of Developmental Biology, University of Science and Culture, Tehran, Iran
}

${ }^{*}$ Corresponding author: Sadeq Vallian, Division of Genetics, Department of Biology, Faculty of Science, University of Isfahan, Isfahan, Iran. Tel/Fax: +983137932456, E-mail: svallian@sci.ui.ac.ir

${ }^{* *}$ Co-Corresponding authors: Mohammad Hossein Nasr-Esfahani, Department of Cellular Biotechnology, Cell Sciences Research Center, Royan Institute for Biotechnology, ACECR, Isfahan, Iran. Tel: +98-3195015680, Fax: +98-3195015687, E-mail: mh.nasr-esfahani@RoyanInstitute.org

Hossein Baharvand, Department of Stem Cells and Developmental Biology, Cell Sciences Research Center, Royan Institute for Stem Cell Biology and

Technology, ACECR, Tehran, Iran. Tel: +98-2122306485, Fax: +98-2122310406, E-mail: Baharvand@RoyanInstitute.org

Received: August 19, 2015; Revised: December 10, 2015; Accepted: March 05, 2016

Background: Promyelocytic leukemia protein (PML) is a tumor suppressor protein that is involved in myeloid cell differentiation in response to retinoic acid (RA). In addition, RA acts as a natural morphogen in neural development.

Objectives: This study aimed to examine $P M L$ gene expression in different stages of in vitro neural differentiation of NT2 cells, and to investigate the possible role of $P M L$ in pluripotency and/or neural development.

Materials and Methods: RA was used as a neural inducer for in vitro neural differentiation of NT2 cells. During this process PML mRNA and protein levels were assessed by quantitative real time RT-PCR (QRT-PCR) and Immunoblotting, respectively. Furthermore bisulfite sequencing PCR (BSP) was used to assess PML promoter methylation in NT2 cells and NT2 derived neuronal precursor cells (NT2.NPCs).

Results: QRT-PCR results showed that, PML had maximum expression with significant differences in NT2 derived neuronal precursor cells relative to NT2 cells and NT2 derived neural cells (NT2.NCs). Numerous isoforms of PML with different intensities appeared in immunoblots of pluripotent NT2 cells, NT2.NPCs, and NT2.NCs. Furthermore, the methylation of the PML promoter in NT2.NCs was 2.6 percent lower than NT2 cell.

Conclusions: The observed differences in $P M L$ expression in different cellular stages possibly could be attributed to the fact that $P M L$ in each developmental state might be involved in different cell signaling machinery and different functions. The appearance of different PML isoforms with more intensity in neural progenitor cells; may suggest apossible role for this protein in neural development.

Keywords: Pluripotent stem cells; Promyelocytic Leukemia; Retinoic acid

\section{Background}

Human $P M L$ gene is located on chromosome 15 (15q) (NC_000015.10; 74287014...74340160). PML protein has several motifs, including an RBCC domain (two zinc finger domains, B boxes, and an $\alpha$-helical coiled-coil motif), nuclear localization signal (NLS), and a serin/prolin rich domain (1). This protein was known as the one of the major components of the promyelocytic leukemia nuclear bodies (PML-NBs). Retinoic acid (RA) acts as a natural morphogen in neu- 
ral development and as a key component of RAdependent myeloid differentiation (2-4). Different pluripotent cell lines were differentiated to mature in vitro neural cells after RA treatment.

$\mathrm{CpG}$ islands (or CG islands) are at least 200 nucleotide regions with more than $50 \%$ GC and $65 \%$ $\mathrm{CpG}$ ratio $(5,6)$. These islands could be considered as indicative of vertebrate genome, which are present in almost $70 \%$ of vertebrate promoters (7). More than $50 \%$ of human genes have these islands at their promoters (8). It has been documented that housekeeping genes, developmental regulatory genes, and tissue specific genes have promoters that possess $\mathrm{CpG}$ islands which are called $\mathrm{CpG}$ promoters (9-12). A number of factors are known to contribute in the control of gene expression in $\mathrm{CpG}$ promoters. These factors include the binding of specific transcription factors such as specific protein one (SP1), the presence of active epigenetic marks and open chromatin structures accessible for transcription machinery $(13,14)$. Among the epigenetic markers, DNA methylation has an important role in the control of gene expression in $\mathrm{CpG}$ promoters. Conversion of cytosine to methyl-cytosine in CpG dinucleotides changes DNA sequence accessibility for active transcription factors, producing compact chromatin structures unsuitable for transcription. DNA methylation as a long term epigenetic mark occurs in $\mathrm{CpG}$ promoters of pluripotent and pluripotent associated genes in stem cells (15).

\section{Objectives}

With regard to the fact that stem cells are a suitable model for in vitro mammalian developmental studies, this study aims to investigate the possible role of $P M L$ in pluripotency and/or neural development after RA treatment. Furthermore, considering DNA methylation as a key factor in the normal development and differentiation process, the DNA methylation pattern of the $P M L$ promoter in pluripotent stem cells and ECderived neural cells (NCs) will also be addressed.

\section{Materials and Methods}

\subsection{Culture and Differentiation of Embryonic carcino- ma Stem Cells (ECSCs)}

The embryonic carcinoma stem cell line (ECSCs;NT2) was cultured in DMEM (Gibco, UK) supplemented with FCS (Gibco) and 2 mM L-glutamine. Cell passage was carried out by treatment with trypsin (Gibco), and cells were seeded in new dishes at a 1:5 ratio. Neural induction of these cells was accom- plished in growth medium supplemented with different inducers and factors over a three-month period. Initially, $2 \times 10^{4}$ cells $/ \mathrm{cm}^{2}$ were seeded in adherent tissue culture dishes in the presence of $10 \mu \mathrm{M}$ RA for a month. The resultant compact neuro epithelial cells were dissociated by trypsin and seeded in a 3:7 ratio in new adherent culture dishes in the presence of $1 \mu \mathrm{M}$ cytosine arabinosin (Sigma, USA) only for the first week of this period, $10 \mu \mathrm{M}$ fluorodeoxyuridine (Sigma, USA) and $10 \mu \mathrm{M}$ uridine for a month. For neural maturation, the apparent cell aggregates were mechanically dissociated by hitting to the side of the tissue culture dish. Dislodged aggregates were seeded in poly-D-lysine (Sigma)-coated dishes in the presence of $1 \mu \mathrm{M}$ cytosine ara binosin (for the first week) and $10 \mu \mathrm{M}$ fluorodeoxyuridine (16).

\subsection{RNA Isolation and Quantitative Real-time PCR}

Total RNA was extracted from three different stages of the neural differentiation process of NT2 cells by TRI reagent (Sigma, USA). Samples were treated with DNaseI (Fermentas, Germany) to remove contaminating genomic DNA. cDNA synthesis was carried out using the Revert Aid First Strand cDNA Synthesis Kit and random hexamer primers (ThermoScientific). Real time PCR was performed with an Applied Biosystems (version 2.1) thermal cycler. Each PCR reaction was performed in triplicate using SYBR Green PCR Master Mix (Takara Bio. Inc., Japan), $2.5 \mathrm{pm}$ of each primer and $25 \mathrm{ng}$ cDNA in a final volume of $10 \mu \mathrm{L}$. Real time PCR data were normalized by GAPDH and relative gene expressions were analyzed by the comparative $\mathrm{Ct}$ method, 2- $\Delta \Delta \mathrm{Ct}$ (17). (Table 1) lists the sequence and the corresponding information for the Real-time PCR primers. The experiments were performed three times in three separate cultures in which cells were differentiated from stem cell stages to mature neural cells. Data from three independent repeats were assessed by one-way ANOVA analysis and presented as mean \pm SEM.

\subsection{Immunoblot Analysis}

Protein extracts were prepared from three stages of neural differentiation process of NT2 cells by TRI reagent (Sigma). Solubilized protein fraction of each sample $(30 \mu \mathrm{g})$ was subjected to SDS-PAGE electrophoresis and transferred to a poly vinylidene fluoride (PVDF) membrane. The membrane was soaked in 5\% $(\mathrm{w} / \mathrm{v})$ skim milk as a blocking solution, rabbit anti-PML antibody (0.5 $\mu \mathrm{g} \cdot \mathrm{mL}^{-1}$, Santa Cruz, USA) and mouse anti-ACTIN antibody (4 $\mu$ g.mL-1, Sigma) as first anti- 
Table 1. Primers that were used for gene expression analysis by QRT-PCR

\begin{tabular}{lcccc}
\hline Genes & Forward Primer (5'-3') & Reverse Primer (5'-3') & AT* $^{*}$ & Accession No \\
\hline GAPDH & CCACTCCTCCACCTTTGACG & CCACCACCCTGTTGCTGTAG & $56^{\circ} \mathrm{C}$ & NM_002046.3 \\
POU5F1 & TCTATTTGGGAAGGTATTCAGC & ATTGTTGTCAGCTTCCTCCA & $60^{\circ} \mathrm{C}$ & NM_001173531.1 \\
NANOG & CAGCTACAAACAGGTGAAGAC & TGGTGGTAGGAAGAGTAAAGG & $56^{\circ} \mathrm{C}$ & NM_024865.2 \\
NESTIN & TCCAGGAACGGAAATCAAG & TTCTCTTGTCCCGCAGACTT & $55^{\circ} \mathrm{C}$ & NM_006617.1 \\
PAX6 & CAGCTCGGTGGTGTCTTTG & AGTCGCTACTCTCGGTTTA & $57^{\circ} \mathrm{C}$ & NM_001127612.1 \\
TUJ1 & AAGCCAGCAGTGTCTAAACCC & GGGAGGACGAGGCCATAAATAC & $60^{\circ} \mathrm{C}$ & NM_006086.2 \\
NCAM & CTCGGCCTTTGTGTTTCCAG & TGGCAGGAGATGCCAAAGAT & $57^{\circ} \mathrm{C}$ & NM_181351.3 \\
PML & ACCTCTGGTTTTCTTTGACCTCAAG & GAACTTGCTTTCCCGGTTCAC & $62^{\circ} \mathrm{C}$ & NM_033238.2 \\
PML-E2-E3 & CGCAAGACCAACAACATC & GAACATCCTCGGCAGTAG & $56^{\circ} \mathrm{C}$ & NM_033238.2 \\
\hline
\end{tabular}

*: AT is annealing temperature

bodies followed by horseradish peroxidase (HRP)-conjugated goat anti-mouse IgG (Dako, Denmark) and HRP-conjugated goat anti-rabbit IgG (Santa Cruz) as secondary antibodies. HRP-conjugated IgGs bound to each protein band were visualized by an Amersham ECL advance Western blotting detection kit (GE Healthcare, Amersham Bioscience, UK).

\subsection{DNA Extraction and Bisulfite Treatment}

Genomic DNA of the NT2 cell line and NT2 derived neural cells (NT2.NCs) was extracted by the DN easy ${ }^{\circledR}$ Blood and Tissue Kit (Qiagen, Germany). Complete bisulfite conversion and DNA clean-up for methylation analysis was performed by the EpiTect ${ }^{\circledR}$ Bisulfite Kit (Qiagen, Germany).

\subsection{Primer Design and Bisulfite Sequencing PCR}

Meth Primer online software was chosen for primer design (www.urogene.org/MethPrimer). The primers used for amplification of PML were as: Forward, TTTTGTAGTTTTGTTTTATTTTTTT and reverse, ATTA ACTAAATCCCTTAAACTATCC. The annealing temperature was $57^{\circ} \mathrm{C}$ (Table 2). The PCR master mix was prepared according to Herman et al. with some modifications (18). Briefly, $300 \mathrm{ng}$ of each forward and reverse primers was used for PCR reactions with $1 \mu \mathrm{L}$ bisulfite modified DNA, 1×ammonium sulfate (AMS) buffer (Cina Clone, Iran), $6 \mathrm{mM} \mathrm{MgCl} 2$ (Cinna Gen, Iran), 1.25 mM dNTP (Cinna Gen, Iran), 0.6 U S-mar Taq (Cinna Gen) and $10 \mathrm{mM}$ 2-mercaptoethanol (Sigma) in a $25 \mu \mathrm{L}$ reaction volume. PCR reaction was carried out in an Eppendorf Master cycler gradient thermal cycler with the following program: $95^{\circ} \mathrm{C}$ for $10 \mathrm{~min}, 35$ cycles at $95^{\circ} \mathrm{C}$ for $30 \mathrm{sec}, 58^{\circ} \mathrm{C}$ for $30 \mathrm{sec}$, and $72^{\circ} \mathrm{C}$ for $1 \mathrm{~min}$, followed by a final extension at $72^{\circ} \mathrm{C}$ for $10 \mathrm{~min}$. PCR products were subcloned into a $\mathrm{pTZ} 57 \mathrm{R} / \mathrm{T}$ cloning vec- tor (InsTAclone ${ }^{\mathrm{TM}}$ PCR Cloning Kit, Fermentas, Germany) according to the manufacturer's protocol. Ligated vectors were transferred into the DH5 $\alpha$ strain of E. coli and grown colonies were selected by PCR analysis through both M13 and reverse sequencing primers. For each group, plasmids from 10 positive colones were extracted by Qiaprep ${ }^{\circledR}$ Spin Miniprep Kit (Qiagen, Germany) and sequenced using standard M13 primers. The obtained sequences were analyzed with bisulfite sequencing DNA methylation analysis (BISMA) online software that defines the methylation pattern of the $P M L$ promoter (19). Using the software, parameters like lower threshold conversion rate and lower threshold sequence identity were chosen at $90 \%$. The upper threshold of $\mathrm{N}$-sites at the cytosine positions and the upper threshold insertions/deletions were chosen by $20 \%$.

\section{Results}

4.1. Formation of Neural Precursor Cells (NPCs) and Neural Cells (NCs) from NT2 Cells

NT2 cells were differentiated in three stages over a three-month process (Figure 1A). Three or four angle NT2 cells (3 or 4 angle) with short and fine processes grew as adherent cell monolayer (Figure 1B). In culture medium that contained RA, NT2 cells propagated as blast slime cells such as neuroepithelial cells. As shown in (Figure 1C), these blast cells formed rosettelike structures in the background of NT2 cells. These heterogenic cultures were treated with trypsin and recultured in medium that contained mitotic inhibitors. After culture, the neuronal precursor cells (NT2.NPCs) appeared as shining clumps with large processes in the background of non-neuronal cells (Figure 1D). NT2 derived neural cells (NT2.NCs), 
which were pure single neurons or clumps of mature neurons (MN) with numerous neurites formed after one month culture of NT2.NPCs in the presence of mitotic inhibitors (Figure 1E).

\subsection{Gene Expression Pattern of NT2 Cells Changes Alongside Neural Differentiation}

In NT2 cells, expressions of OCT4 and NANOG decreased in NT2.NPCs and NT2.NCs (Figure 2A). Expressions of NESTIN and PAX6, two neural precursor markers, were significant in NT2.NPCs. However, there was no significant expression of these markers in NT2 cells and in NT2.NCs (Figure 2B). Expressions of TUJ1 and NCAM, two neuronal markers, was not detected in NT2 cells (Figure 2C). In contrast, increased expressions of these markers were observed in NT2.NPCs and NT2.NCs. This level of expression was significantly higher compared to NT2 cells (Figure 2C).

In parallel with these gene expression assessments, a very slight level of $P M L$ expression was noted in NT2 cells (Figure 3A). Following RA treatment and neural induction, higher levels of $P M L$ and $P M L$ E2E3 expressions were detected in NT2.NPCs compared
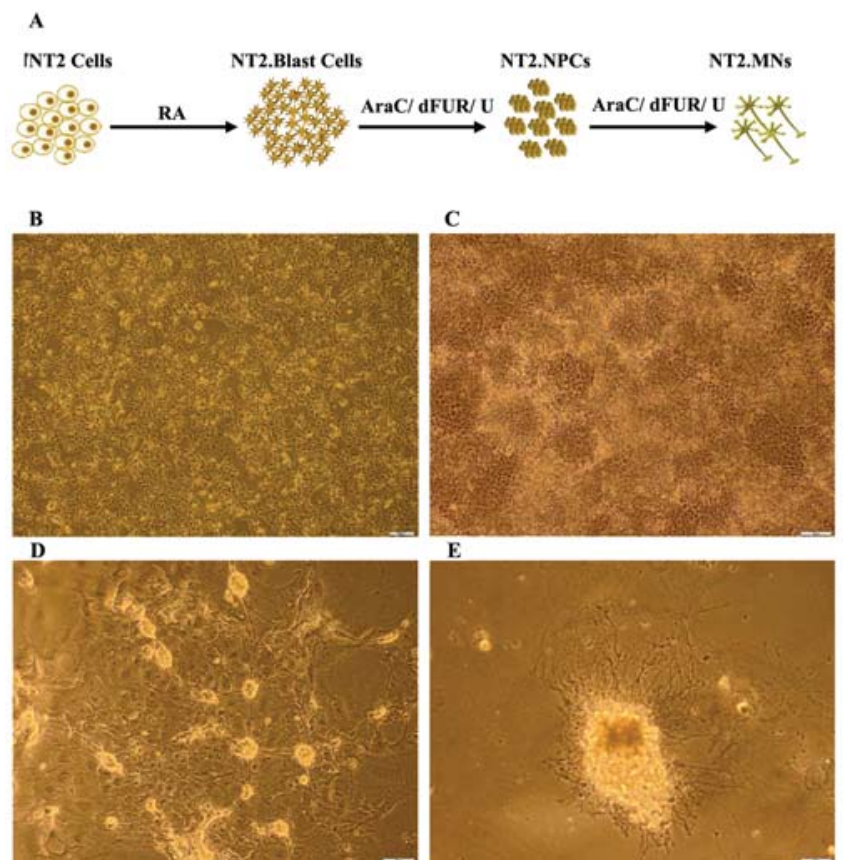

Figure 1. The identity of NT2 cells changed following retinoic acid (RA) treatment. A: Schematic diagram of neural induction in NT2 cells by sequential RA and mitotic inhibitor treatment. B: NT2 cell line in pluripotent state grew as cell monolayer. C: In the presence of RA, the cells form rosette-like structures contained blast cells. D: NT2.NPCs formed in the presence of mitotic inhibitors. E. Pure mature neurons (MN) appeared after three months of NT2 neural induction to NT2 cells. However and at the end of the differentiation process, $P M L$ and $P M L$ E2-E3 expressions in NT2. NCs decreased with a level similar to NT2 cells (Figure 3A). Immunoblotting was performed on the extracts of NT2 cells, NT2.NPCs and NT2.NCs showed the presence of different PML isoforms. On the extracts of the NT2 cells, seven bands in the range of 70-200 kDa were observed. In NT2.NPCs, the same pattern with increased intensity was detected. However, in NT2.NCs, there was no $170 \mathrm{kDa}$ PML
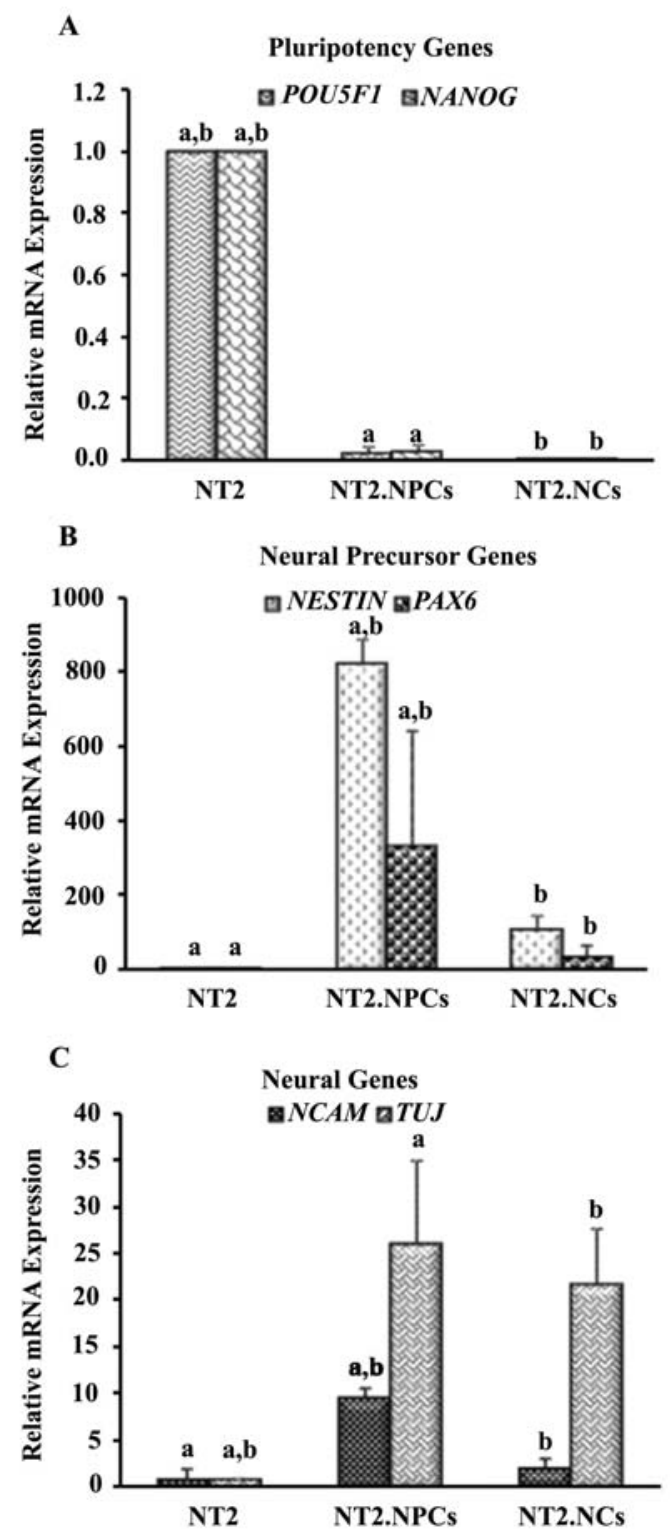

Figure 2. Gene expression of NT2 cells changed following retinoic acid (RA) treatment. A: Relative expression level of pluripotency genes decreased in neural cells. B: Relative expression level of neural precursor genes was higher in NT2.NPCs. C: Neural genes had maximum expression in NT2.NCs. Means with similar letters are significantly different (Tukey, LSD, $\mathrm{p}<0.05$ ) 
isoform. Two sharp bands within $130 \mathrm{kDa}$ were observed and also the intensity of the other bands with lower molecular weights was attenuated (Figure 3B).

\subsection{Bisulfite Sequencing PCR of the PML Promoter in} NT2 and NT2.NPCs

The human $P M L$ promoter region from $-809 /+800$ relative to the transcription start site (7428620574287814) was imported as an input sequence in Meth Primer software (20). The promoter sequence was submitted in the main page of the software and primers were designed for BSP. Bioinformatic analysis showed one $\mathrm{CpG}$ island with $109 \mathrm{bp}$ at $865-973$ region in a TATA-less $P M L$ promoter (Figure 4A). This software, introduced a pair of primer suitable for BSP in upstream of the $\mathrm{CpG}$ island (Figure $3 \mathrm{~A}$ and $4 \mathrm{~B}$ ). DNA samples extracted from NT2 and NT2.NPCs were subjected to BSP. PCR products were subcloned in a pTZ57R/T cloning vector and final positive clones used as the template for sequencing. Sequencing analysis by BISMA online software showed differences in NT2 cells and NT2,NPCs on the average methylation for each $\mathrm{CpG}$ site, the number of $\mathrm{CpG}$ sites, percent of DNA methylation, the average methylation over all sequences and the average methylation for each clone (Figure 4C and D). In NT2 derived clones, 4 clones out of 10 clones were methylated. These clones had only one methylated $\mathrm{CpG}$ in the third or fourth $\mathrm{CpG}$ dinucleotide of the predicted $\mathrm{CpG}$ isl and. Methylated CpGs were located in the 48 and 67 region of the $\mathrm{CpG}$ island and methylated 14.3 and 28.6 percent, respectively (Figure 4C). In NT2.NPCs derived clones only one $\mathrm{CpG}$ in the 198 region of the predicted $\mathrm{CpG}$ island, with 14.3 percent, was methylated (Figure 4D).

\section{Discussion}

The role of PML in granulocyte differentiation has been previously reported (21). However, to date, there is no report on the role of PML protein and methylation of its gene promoter in nervous system development and differentiation in human (22). Therefore, to understand the eventual role of this protein in neural development,NT2 pluripotent cell line were treated with RA as an accepted model for in vitro neural differentiation $(3,23)$. After RA treatment of pluripotent stem cells, NPCs with different patterns and morphologies were observed. Following adherent culture of NPCs, at the end of the neural differentiation process, NPCs were potentiated and changed to MNs with neuronal processes. It has been proved that in pluripotent stem cell lines parallel to neural differentiation process, expression of pluripotency genes such as OCT4 and NANOG decreased in stem cells and the expression of neural genes including MAP2 and TUJ1 increased in mature neuronal cells (24-28). This phenomen, similar to other pluripotent stem cell lines, was observed in NT2 and NT2 derived neural cells and confirmed the proposed neural differentiation process. Assessment of $P M L$ expression in different stages of neural differentiation showed that $P M L$ had a minimum of expression in pluripotent NT2 cells and reached maximum expression after RA treatment in the neural precursor cells. In embryonic carcinoma cells, PML-NBs were shown to be involved in OCT4 expression and high level of $P M L$ expression was expected in NT2 cells. While higher $P M L$ expression in invasive and proliferative cells of liver carcinoma in contrast to lower PML expression in proliferative cells of lung cancer has been reported (29). Therefore, in the present study, since NT2 cells have been derived from
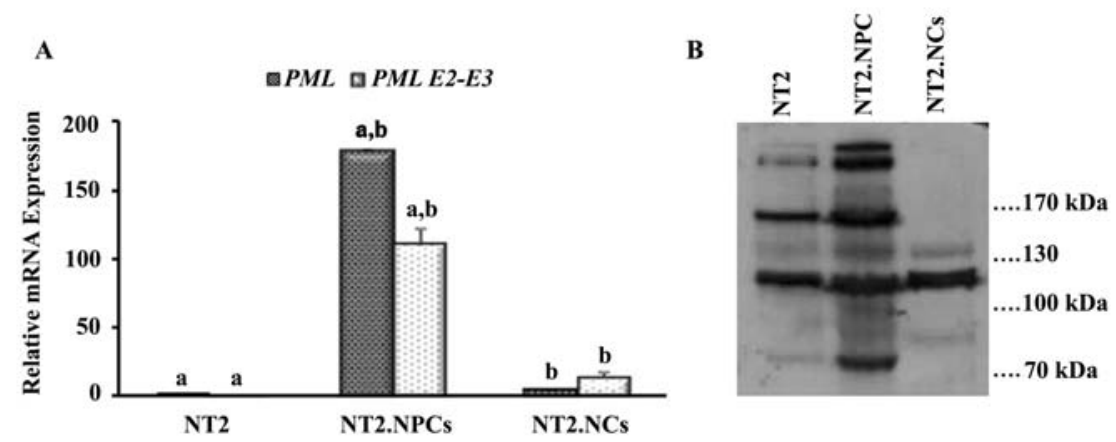

Figure 3. Assessment of PML expression in mRNA and protein level during NT2 cells neural differentiation. A: Relative PML expression was higher in NT2.NPCs than in NT2 and NT2.NCs. Means with similar letters are significantly different (Tukey's, LSD, $\mathrm{p}<0.05)$. B: Different PML isoforms with different intensities were detected in immunoblot analysis of PML in NT2 cells, NT2.NPCs and NT2.NCs 


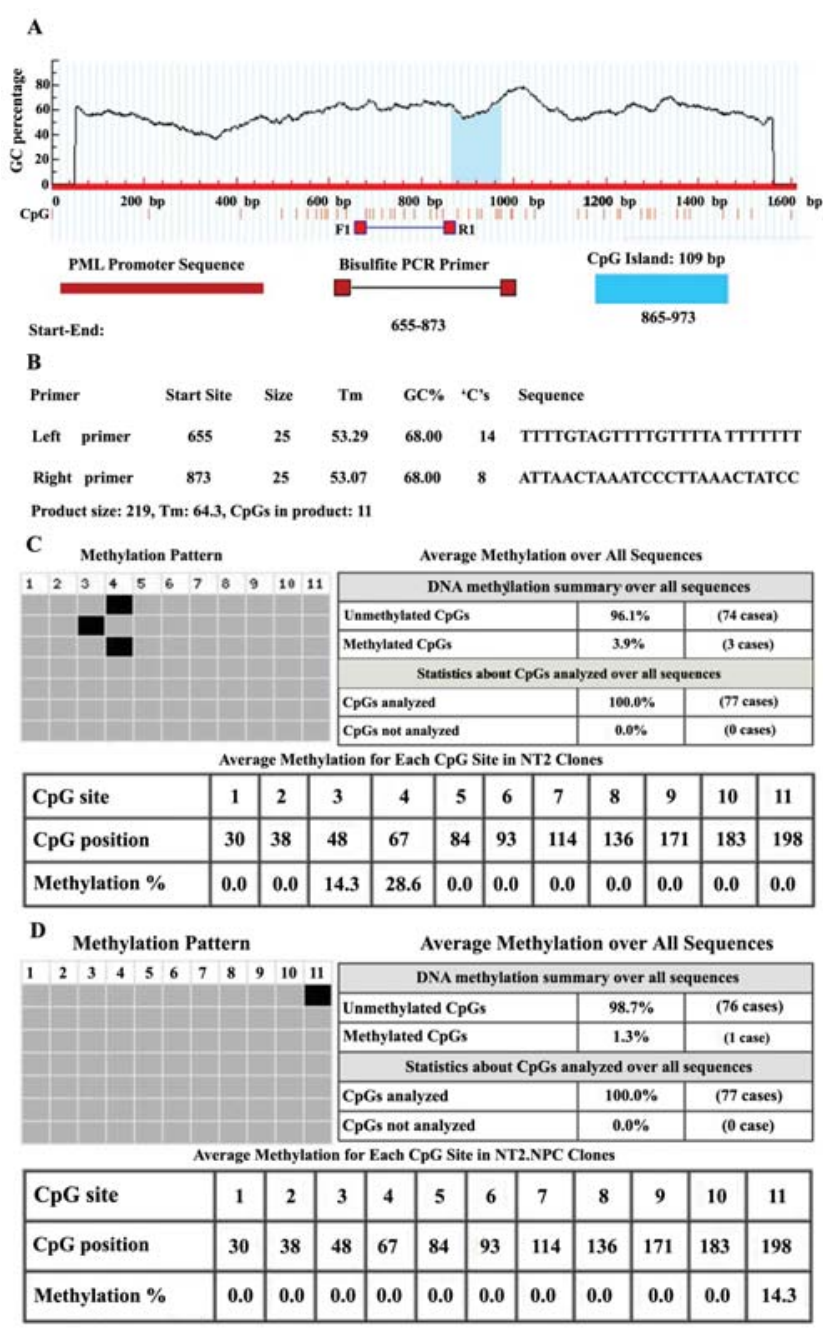

Figure 4. Analysis of $P M L$ promoter by Methprimer and BISMA online software programs. A: In a shematic representation of Methprimer software, the position of the $\mathrm{CpG}$ island with 109 base pair and the position of suitable BSP specific primer pair in upstream region of the $\mathrm{CpG}$ island is shown. B: Characteristics included the BSP specific primer sequences, melting temperatures and product length with the number of $\mathrm{CpG}$ nucleotides has been shown for the proposed PCR product. C: Results of BISMA online software analysis of PML promoter in NT2 cells showed one methylated CpG in three clones. D: Results of BISMA online software in NT2.NPCs showed one methylated CpG in only one clone

metastatic lung cancer, low $P M L$ expression in NT2 cells might explain their metastatic nature of origin (lung) (30).

Furthermore, it is important to note that although NT2 cells are pluripotent cells, they have the ability to generate all three cell types of the central nervous system. Therefore, they are considered as neural progenitor cells (30). In view of the presence of $P M L$ expression in proliferating neural progenitor cells of the developing mouse neocortex, it is belived that $P M L$ probably involve in the proliferation of neural progenitor cells (31). In these cells, PML controls protein phosphatasemediated dephosphorylation of retinoblastoma tumor suppressor protein (32).

In human cells, alternative splicing in the carboxyterminal domains of $P M L$ gene has generated varoiuse PML isoforms. Regarding to this subject, assessment of the relative mRNA expression level of the first and longest isoform carried out with one primer pair specific to the 8a-9 exon junction. Since there is no specific exon junction in other isoforms (there is a similar sequence), evaluation of all $P M L$ isoforms specifically is impossible. Therefore, one primer pair complementary to the second and third exon junction, called $P M L$ E2-E3, was used for assessment of the relative mRNA expression level of other $P M L$ isoforms (33). The results indicated that the relative mRNA expression of the $P M L I$ and all other $P M L$ isoforms during neural differentiation in NT2 cells was similar in the three studied stages. This could be explained by the fact that since different isoforms have a different localization and probably different function in transfected fibroblast cells, the observed similar expression level could be possible and could not reject specific function for each isoform. This may indicate that all PML isoforms may cooperatively involve in different cellular activity.

Our immunoblot showed several bands representative of different PML isoforms in different stages of NT2 neural differentiation process. Interestingly, similar to QRT-PCR results (see above), the number and intensity of bands that express the presence of different PML isoforms were higher in NT2.NPCs. It has been reported that post-translational modifications like sentrinization result in different PML migrating isoforms $(34,35)$. Therefore these bands may relate to different PML isoforms and or different forms of one isoforms of PML. There is no report on the expression pattern of PML isoforms in stem cells, nor in NCs derived from in vivo or in vitro embryos or cultures. In one report from Hsu and Everett only the increment of PML and its presumed SUMO-1 modified form after RA treatment has been reported in neuron-like cells obtained from NT2 cells (36). It has been shown that PML in neocortex progenitor cells controls neural differentiation; and in cortex neurons by Glu1A inhibition regulates synaptic strength (37). Therefore, the presence of several protein bands in both pluripotent cells and NCs may suggest that this protein, in addition to its possible role in pluripotency and proliferation, regulates synapse plasticity that has previously been reported in cortex neurons. Obviously, in view of dif- 
ferent post-translational modifications of the PML protein in different situations (38), further studies are required to fully decipher the nature of the PML protein bands and determine which is active in pluripotency and/or neural development.

Despite the presence of extensive reports on the different aspects of $P M L$ function, the importance of the methylation pattern of the $P M L$ promoter is unclear. Therefore, in the next step, the methylation pattern of the $P M L$ gene promoter was investigated in NT2 and mature neuronal cells. The process of neural differentiation in NT2 cells usually lasts three months and results in pure neuron cells. However, due to the long term culture of final neurons, it was technically impossible to prepare suitable bisulfite treated DNA for bisulfite sequencing of NT2-NCs. Therefore, the methylation pattern of the PML promoter in NT2 cells as pluripotent stem cell sand NT2-NPCs as neural cells examined. Our results showed that in both NT2 cells and NT2-NPCs, the PML promoter was differentially methylated. The methylation pattern of the PML promoter differed in NT2 cells and NT2-NPCs, but in both cell types the PML promoter showed a low degree of methylation (3.9 pecent in NT2 cells and 1.3 percent in NT2.NCs). Since NT2-NPCs and NT2-NCs were from a neural lineage, therefore, it was expected that these two cell types would have similar methylation patterns. The observed differential methylation pattern could be discussed in relation to the expression pattern of $P M L$ in these cells.

As mentioned above, quantitative RT-PCR (QRTPCR) results showed that relative $P M L$ expression in NT2.NPCs was 30-fold more than NT2 cells. However according to our data, it is believed that the DNA methylation pattern could not solely describe the observed difference. It is possible that other factors may have contributed in this phenomenon. Further studies are required to determine if the difference in $P M L$ expression during neural differentiation of NT2 cells is related to differential $\mathrm{CpG}$ methylation or whether other factors regulate PML expression.

\section{Acknowledgements}

This study was funded by a grant provided from Royan Institute.

\section{References}

1. Freemont PS, Hanson IM, Trowsdale J. A novel gysteine-rich sequence motif. Cell 1991;64(3):483-484.

2. Engberg N, Kahn M, Petersen DR, Hansson M, Serup P. Retinoic acid synthesis promotes development of neural progenitors from mouse embryonic stem cells by suppressing endogenous, Wnt-dependent nodal signaling. Stem Cells 2010;28(9):1498-1509. DOI: 10.1002/stem.479

3. Rhinn M, Dollé P. Retinoic acid signalling during development. Development 2012;139(5):843-858. DOI: 0.1242/dev.065938.

4. Marlétaz F, Holland LZ, Laudet V, Schubert M. Retinoic acid signaling and the evolution of chordates. Int $J$ Biol Sci. 2006;2(2):38-47.

5. Sharif J, Endo TA, Toyoda T, Koseki H. Divergence of CpG island promoters: a consequence or cause of evolution? Dev Growth Differ. 2010;52(6):545-554. DOI: 10.1111/j.1440169X.2010.01193.X

6. Takai D, Jones PA. Comprehensive analysis of $\mathrm{CpG}$ islands in human chromosomes 21 and 22. PNAS. 2002;99(6):37403745 .

7. Saxonov S, Berg P, Brutlag DL. A genome-wide analysis of $\mathrm{CpG}$ dinucleotides in the human genome distinguishes two distinct classes of promoters. Proc Natl Acad Sci USA. 2006;103(5):1412-1417.

8. Vavouri T, Lehner B. Human genes with $\mathrm{CpG}$ island promoters have a distinct transcription-associated chromatin organization. Genome Biol. 2012;13(11):R110. DOI: 10.1186/gb-2012-13-11r110

9. Ponger L, Duret L, Mouchiroud D. Determinants of $\mathrm{CpG}$ islands: expression in early embryo and isochore structure. Genome Res. 2001;11(11):1854-1860.

10. Zhu J, He F, Hu S, Yu J. On the nature of human housekeeping genes. Trends Genet. 2008;24(10):481-484. DOI: 10.1016/ j.tig.2008.08.004.Epub 2008 Sep 9

11. Ramsköld D, Wang ET, Burge CB, Sandberg R. An abundance of ubiquitously expressed genes revealed by tissue transcriptome sequence data. PloS comp Biol. 2009;5(12):e1000598. DOI: 10.1371/journal.pcbi.1000598. Epub 2009 Dec 11

12. Larsen F, Gundersen G, Lopez R, Prydz H. CpG islands as gene markers in the human genome. Genomics 1992;13(4):1095-1097.

13. Butler JE, Kadonaga JT. The RNA polymerase II core promoter: a key component in the regulation of gene expression. Genes Dev. 2002;16(20):2583-2592.

14. Deaton AM, Bird A. CpG islands and the regulation of transcription. Genes Dev. 2011;25(10):1010-1022. DOI: 10.1101/ gad.2037511

15. Reik W. Stability and flexibility of epigenetic gene regulation in mammalian development. Nature 2007;447(7143):425-432.

16. Horrocks GM, Lauder L, Stewart R, Przyborski S. Formation of neurospheres from human embryonal carcinoma stem cells. Biochem Biophys Res Commun. 2003;304(2):411-416.

17. Schmittgen TD, Livak KJ. Analyzing real-time PCR data by the comparative CT method. Nat Protoc. 2008;3(6):1101-1108.

18. Herman JG, Graff JR, Myöhänen S, Nelkin BD, Baylin SB. Methylation-specific PCR: a novel PCR assay for methylation status of CpG islands. Proc Natl Acad Sci USA. 1996;93(18):9821-9826.

19. Rohde C, Zhang Y, Reinhardt R, Jeltsch A. BISMA-Fast and accurate bisulfite sequencing data analysis of individual clones from unique and repetitive sequences. $B M C$ Bioinformatics. 2010;11(1):230. DOI: 10.1186/1471-210511-230

20. Stadler M, Chelbi-Alix MK, Koken M, Venturini L, Lee C, Saib A, et al. Transcriptional induction of the $P M L$ growth 
suppressor gene by interferons is mediated through an ISRE and a GAS element. Oncogene 1995;11(12):2565-2573.

21. Khalfin-Rabinovich Y, Weinstein A, Levi B-Z. PML is a key component for the differentiation of myeloid progenitor cells to macrophages. Int Immunol. 2011;23(4):287-296. DIO: 10.1093/intimm/dxr004.Epub 2011 Mar 22

22. Korb E, Finkbeiner S. PML in the brain: from development to degeneration. Front Oncol. 2013;3. DOI: 10.3389/fonc. 2013.00242

23. Gudas LJ, Wagner JA. Retinoids regulate stem cell differentiation. J Cell Physio. 2011;226(2):322-330. DOI: 10.1002/jcp. 22417

24. Draper JS, Pigott C, Thomson JA, Andrews PW. Surface antigens of human embryonic stem cells: changes upon differentiation in culture. J Anat. 2002;200(3):249-258.

25. Bhattacharya B, Cai J, Luo Y, Miura T, Mejido J, Brimble SN, et al. Comparison of the gene expression profile of undifferentiated human embryonic stem cell lines and differentiating embryoid bodies. BMC Dev Biol. 2005;5(1):22.

26. Schulz TC, Palmarini GM, Noggle SA, Weiler DA, Mitalipova MM, Condie BG. Directed neuronal differentiation of human embryonic stem cells. BMC Neurosci. 2003;4(1):27.

27. Baharvand $\mathrm{H}$, Mehrjardi N, Hatami M, Kiani S, Rao M, Haghighi M. Neural differentiation from human embryonic stem cells in a defined adherent culture condition. Int J Dev Biol. 2007;51(5):371-378.

28. Bain G, Kitchens D, Yao M, Huettner JE, Gottlieb DI. Embryonic stem cells express neuronal properties in vitro. Dev Biol. 1995;168(2):342-357.

29. Yu E, Choi EK, Kim CJ. Expression of promyelocytic leukemia protein increases during the differentiation of human neuroblastoma cells. Virchows Arch. 2003;442(3):278-283.
30. Pleasure S, Lee VY. NTera 2 cells: a human cell line which displays characteristics expected of a human committed neuronal progenitor cell. J Nuro Res. 1993;35(6):585-602.

31. Regad T, Bellodi C, Nicotera P, Salomoni P. The tumor suppressor Pml regulates cell fate in the developing neocortex. Nat Neurosci. 2009;12(2):132-140. DOI: 10.1038/nn.2251.Epub 2009 Jan 11

32. Guan D, Kao HY. The function, regulation and therapeutic implications of the tumor suppressor protein, PML. Cell Biosci. 2015;5(1):1. DOI: 10.1186/s13578-015-0051-9.e Collection 2015

33. Condemine W, Takahashi Y, Zhu J, Puvion-Dutilleul F, Guegan S, Janin A. Characterization of endogenous human promyelocytic leukemia isoforms. Cancer Res. 2006;66(12):6192-6198.

34. Kamitani T, Kito K, Nguyen HP, Wada H, Fukuda-Kamitani T, Yeh ET. Identification of three major sentrinization sites in PML. J Biol Chem. 1998;273(41):26675-26682.

35. Zhong S, Müller S, Ronchetti S, Freemont PS, Dejean A, Pandolfi PP. Role of SUMO-1-modified PML in nuclear body formation. Blood 2000;95(9):2748-2752.

36. Hsu W-L, Everett RD. Human neuron-committed teratocarcinoma NT2 cell line has abnormal ND10 structures and is poorly infected by herpes simplex virus type $1 . J$ Virol. 2001;75(8):3819-383.

37. Korb E, Wilkinson CL, Delgado RN, Lovero KL, Finkbeiner S. Arc in the nucleus regulates PML-dependent GluA1 transcription and homeostatic plasticity. Nat Neurosci. 2013;16(7):874-883. DOI: 10.1038/nn.3429. Epub 2013 Jun 9.

38. Cheng X, Kao HY. Post-translational modifications of PML: consequences and implications. Front Oncol. 2012;2:210. DOI: 10.3389/fonc.2012.00210.eCollection 2012 